\title{
TRUNK MOTOR CONTROL IN PEOPLE WITH LOW BACK PAIN DURING GOAL-DIRECTED SAGITTAL TRUNK MOVEMENTS
}

D. Jiménez-Grande, A.M. De Nunzio, A.M. Alsubaie

Centre of Precision Rehabilitation for Spinal Pain (CPR Spine), School of Sport, Exercise and Rehabilitation Sciences, University of Birmingham, UK

\section{Introduction}

- Repetitive sagittal trunk movements represent an occupational risk factor leading to cumulative spinal loading even when preformed with no or small weight lift [1]

- These cumulative spinal loads especially from high pace repetitive motion can be associated with the development of low back pain (LBP) [2].

- A possible mechanism causing an increased loading effect in LBP population relates to impaired trunk motor control in form of reduced precision of movement [3].

- Another component of motor control impairment as a result of LBP is altered muscle recruitment patterns, in form of increased coactivation and reduced deep muscle activity [4].

\section{Aim}

- Explore the effect of varying speed of motion on the kinematic variability of movement as well as the muscle recruitment patterns during repetitive goal-directed sagittal trunk movements in LBP people compared to asymptomatic control.

\section{Methods}

Seventeen healthy participants (control group, $29.8 \pm 4$ years), and fifteen with chronic non-specific low back pain (LBP group, $37.2 \pm 9$ years) were enrolled in this study. The average pain intensity for the LBP group on the visual analog scale (VAS) was 5.2/10 \pm 2.7 .

Trunk motion was acquired using eight optoelectronic infrared cameras (BTS Bioengineering, Italy) and 17 reflective markers to construct 3D kinematic data of the trunk. Six wireless electromyography (EMG) probes placed bilaterally on erector spinae (ES), latissimus dorsi (LD), and obliques externus abdominis (EOA) muscles.

Subjects were instructed to perform 12 continuous cycles from $20^{\circ}$ extension to $50^{\circ}$ trunk flexion at two different speed levels: $30^{\circ} / \mathrm{sec}$ (slow), $50^{\circ} / \mathrm{sec}$ (high).

One-way analysis of variance (ANOVA) was conducted to detect any significance difference between the two groups in term of temporal differences using crosscorrelation measures to detect any lag, the Kinematic variability based on standard deviation measures and Co-Contraction Index (\%).

\section{Results}

ANOVA results revealed significant temporal differences between the two groups when following the feedback in high speed $(p=0.03)$ as well as slow speed $(p=0.00)$ motion. The control group were anticipating the feedback in high speed motion by $(0.51 \pm 0.48$ second $)$ and by $(0.63 \pm 0.78$ second) in slow motion speed.
- The LBP group were anticipating the feedback only in high speed motion by $(0.10 \pm 0.30$ second) while in slow motion LBP participants had a delayed motion compared to the feedback signal by $(0.18 \pm 0.32$ second) (Fig.1).

- No significant difference was found in kinematic variability between both groups. However, Moderate to high linear correlation was detected between the variability of lumber spine segment and $F A B Q$ score during high ( $r=0.35)$ and slow ( $r=0.50)$ motion (Fig. 2).

- The percentage of the co-contraction index for the LBP group was higher than the control group (Table 1). This was statistically significant for the right-side muscles during both high and slow motion $(p=0.00)$.


Table 1: co-contraction index.

\begin{tabular}{|c|c|c|c|c|}
\hline Speed & Side & LBP Group & Control Group & P value \\
\hline \multirow{2}{*}{$\begin{array}{c}\text { High speed } \\
50^{\circ} / \mathrm{sec}\end{array}$} & Right side & $74.66 \%$ & $70.15 \%$ & $P=0.00$ \\
\hline & Left side & $74.13 \%$ & $72.32 \%$ & $P=0.18$ \\
\hline \multirow{2}{*}{$\begin{array}{c}\text { Slow speed } \\
30^{\circ} / \mathrm{sec}\end{array}$} & Right side & $89.92 \%$ & $86.48 \%$ & $P=0.00$ \\
\hline & Left side & $90.25 \%$ & $89.39 \%$ & $P=0.34$ \\
\hline
\end{tabular}

\section{Conclusion}

- LBP patients tend to be slower in learning motor adaptive behaviors. Furthermore, the newly learned behavior could be associated with adverse consequences such as more rigid and less accurate movement.

- No significant difference was detected in kinematic variability between both groups. However, LBP patients with higher fear of movement tend to move in a more variable way.

References

.

Coenen, Kingma et al. (2013) Journal of occupational rehabilitation 23(1): 11-18.

Sung, Abraham et al. (2015) The spine journal official journal of the North American Spine Society 15(8):

van Dieën (2017) Exercise \& Sport Sciences Reviews 45(4): 223-230 